\title{
Forma atípica de enfermedad por arañazo de gato: Compromiso óseo en dos pacientes pediátricos detecta- do mediante cintigrafía ósea. Revisión de la literatura
}

DAVID LADRÓN DE GUEVARA H. ${ }^{1}$, GABRIEL LOBO S.${ }^{1}$,

MARTA MIRANDA A. ${ }^{2}$, ELBA WU H. ${ }^{3}$, M. ANGÉLICA MUÑOZ G. ${ }^{1}$,

ANDRÉS PÉREZ R. ${ }^{1}$ y CÉSAR JIMÉNEZ J. ${ }^{1}$

\author{
Atypical form of Cat-Scratch Disease: osseous involvement in two children \\ detected by bone scan. Review of literature
}

Cat-Scratch Disease (CSD) is a benign, self-limited illness that occurs after scratch or bite of a cat carrier of Bartonella henselae. It presents as lymphadenopathy near to the inoculation site, and affects in $80 \%$ of cases individual younger that 21 years old. Fewer than $25 \%$ of patients will have the atypical form of the disease, with involvement of organs as liver, spleen, central nervous system and retina. Osteomyelitis has been claimed in old series as rare, although more recent publications suggest a higher incidence. Axial skeleton is the most frequently involved, especially pelvis and dorsal and lumbar spine. Skeletal lesions can be only one or multiple. Lesions not always cause pain and they can appear weeks or months after the beginning of symptoms. We describe two girls with skeletal involvement, positive serology for $B$. henselae and complete recovery. One of them (12 yr) had fever and abdominal pain, with a hypodense lesion in liver and multifocal osteomyelitis in spine and pelvis. The other one (10 yr) had sub-maxillary adenopathy and one painless lesion in iliac crest, which was evident in bone scan (BS), without fever or worsening of general condition. We recommend the regular use of BS in patients with CSD due to poor sensitivity of radiography in detection of bone envolvement and because these lesions can be multifocal and painless in some cases.

Key words: Cat-Scratch Disease; Bartonella henselae; Osseous involvement; Osteomyelitis; Bone scan.

\section{Introducción}

El compromiso del aparato locomotor siempre significa un reto para el médico. Sus causas son diversas: desde traumáticas, infecciosas e inflamatorias, a tumorales. Sus manifestaciones clínicas son variables, dependiendo de la edad del paciente y su etiología. Con este espectro tan diverso, frente a un niño con síntomas y signos músculo-esqueléticos, nuestro objetivo será siempre descartar un proceso infeccioso o tumoral. Así la patología del aparato locomotor de un recién nacido o un lactante, obligará a descartar como primera posibilidad una osteo-artritis séptica, la cual habitualmente se desarrollará en el contexto de un cuadro infeccioso sistémico ${ }^{1}$. Es diferente en el pre-escolar y escolar, donde surge una gran gama de patologías; los cuadros infecciosos en este grupo etario ya no ocupan el primer lugar y las manifestaciones pueden estar dadas tanto por focalización del microorganismo en el esqueleto, así como ser una respuesta reactiva a la presencia del mismo en el organismo. En ellos, el cuadro clínico puede expresarse como un síndrome febril, con o sin síntomas dolorosos del aparato locomotor. Actualmente, a

\footnotetext{
Hospital San Juan de Dios:

Unidad de Medicina Nuclear.

Departamento de Reumatología.

Departamento de Pediatría.
} 
la lista de bacterias causantes de osteomielitis o artritis, deberemos agregar Bartonella henselae $e^{2,3}$.

Dicha bacteria fue identificada por Wear et al en $1983^{4}$, y es agente causal de patologías como angiomatosis bacilar, endocarditis, peliosis visceral $\mathrm{y}$, en pacientes inmunocompetentes, de la enfermedad por arañazo de gato (EAG) $)^{5-7}$. Esta última es una enfermedad de curso benigno, generalmente autolimitada, transmitida al ser humano por el rasguño o mordisco de un gato portador. En nuestro país ha sido reconocida como una infección emergente ${ }^{8}$. La EAG es una identidad clínica polimórfica, posible de manifestar mediante diversos síndromes, de los cuales la linfoadenitis regional subaguda o crónica, sin compromiso de otros sistemas, es el más frecuente, constituyendo la forma típica de la enfermedad $^{5.9}$. Afecta en $80 \%$ de los casos a individuos bajo 21 años de edad ${ }^{10}$, especialmente escolares y adolescentes, y siempre se asocia a contacto con gatos, aunque algunos autores también han descrito contagio mediado por perros ${ }^{11-13}$ Aproximadamente 10\% de los gatos "mascotas" y $33 \%$ de los "callejeros" portan B. henselae en su sangre, siendo aquellos con menos de un año de vida e infestados con pulgas, los de mayor riesgo ${ }^{5,14}$.

El cuadro clínico se inicia dos a tres semanas después de la exposición al microorganismo y se caracteriza por una linfoadenopatía de curso crónico, en los pacientes con los antecedentes de contacto antes comentados. Un tercio de ellos presenta fiebre y compromiso del estado general. En la evolución pueden existir signos inflamatorios en el sitio de inoculación y adenopatías de ganglios linfáticos en la zona de drenaje del ganglio afectado. Síntomas menos frecuentes son anorexia, pérdida de peso y esplenomegalia. El cuadro es autolimitado en las personas inmunocompetentes.

El diagnóstico de EAG se debe sospechar frente a un cuadro clínico sugerente, sumado al antecedente epidemiológico. Su pesquisa se puede efectuar con tests cutáneos ${ }^{15}$, cuyo uso es inhabitual. En caso de disponer de material de biopsia ganglionar, la tinción de plata de WarthinStarry podría ayudar demostrando la presencia de un bacilo Gram negativo. El cultivo de sangre o tejido es de desarrollo lento, con incubaciones prolongadas (6 semanas). Las pruebas serológicas para detectar tanto anticuerpos como antígenos: IFI, enzimoinmunoensayo (EIA), son los de mayor uso actualmente ${ }^{5,16}$, de acuerdo a lo descrito en la literatura y en nuestro medio. Títulos $>$ de 1/ 256 (IFI) son considerados positivos por el laboratorio empleado en el presente caso. La RPC sólo se utiliza como método de investigación.
No existe consenso respecto a la indicación del tratamiento antimicrobiano dado que en las personas inmunocompetentes, es habitualmente una afección autolimitada; sólo se indica tratamiento en aquellos pacientes inmunocomprometidos que desarrollan una afección de curso inusual.

Las formas atípicas de la EAG corresponden a 5 a $25 \%$ de los $\operatorname{casos}^{5}$, y producen un cuadro sistémico severo, frecuentemente asociado a fiebre alta, malestar general importante, baja de peso y compromiso de órganos como hígado, bazo, SNC, nervios periféricos y retina. La afectación ósea también ha sido descrita en algunos trabajos, y aunque su ocurrencia parece ser ocasional, su frecuencia exacta no ha sido establecida. El amplio espectro de manifestaciones clínicas de la EAG atípica hace que su diagnóstico sea complejo y frecuentemente tardío, debutando clínicamente como cuadros de abdomen agudo $^{17,18}$, síndrome febril prolongado ${ }^{16,19-21}$ neoplá$\operatorname{sicos}^{22-24}$, neurológicos ${ }^{13,21,25,26}$, etc.

El compromiso óseo en la EAG atípica ha sido descrito como lesiones predominantemente líti$\mathrm{cas}^{5}$, pudiendo localizarse tanto en esqueleto axial como apendicular. En su estudio se han empleado técnicas radiológicas y de medicina nuclear, principalmente radiografía, TAC y cintigrama óseo (CO).

Publicamos los hallazgos clínicos, de laboratorio e imagenológicos encontrados en dos pacientes pediátricos internados en el Hospital San Juan de Dios por EAG y compromiso óseo, realizando una revisión de la literatura médica correspondiente.

Caso clínico 1. Adolescente 12 años, sexo femenino, sin antecedentes mórbidos de importancia, que ingresó con cuadro febril asociado a dolor abdominal intenso, con una semana de evolución, sin diarrea ni vómitos. Destacaba leucocitosis de $17.300 / \mathrm{mm}^{3}$ sin desviación a izquierda, PCR: $66 \mathrm{mg} / \mathrm{Lt}$, VHS: $80 \mathrm{~mm} / \mathrm{h}$, sedimento de orina normal. La radiografía de abdomen simple y de tórax no mostraron hallazgos patológicos. La ecotomografía abdominal realizada al tercer día de hospitalización mostró adenopatías en hilio hepático, sin otras alteraciones. Al décimo día la paciente persistía con fiebre hasta $38,5^{\circ} \mathrm{C}$, aunque el dolor abdominal se hizo cada vez menos intenso, apareciendo discreto dolor lumbosacro. Un segundo hemograma reveló 12.900 leucocitos $/ \mathrm{mm}^{3}$ sin desviación a izquierda, PCR: $35 \mathrm{mg} / \mathrm{Lt}$, VHS: $75 \mathrm{~mm} / \mathrm{h}$. Una nueva ecotomografía abdominal mostró adenopatías retroperitoneales y en el hilio hepático. Una 
ecocardiografía dio resultado normal, y tanto la endoscopia digestiva alta como el mielograma mostraron alteraciones inespecíficas. Una TAC abdominal realizada al $17^{\circ}$ día describió una lesión focal hipodensa en el segmento VI hepático de $1 \times 1 \mathrm{~cm}$, y una adenopatía en hilio hepático de $2 \times 2 \mathrm{~cm}$. El cintigrama óseo realizado al día siguiente (dosis: $17 \mathrm{mCi}$ ev de MDP-Tc99m, gammacámara SMV DST XLi doble cabezal) mostró un aumento anormal de captación en los cuerpos vertebrales D11, L1 y L2, y en la articulación sacroilíaca izquierda (Figura 1). Al momento de realizarse este examen la paciente presentaba dolor lumbosacro derecho, y aún se mantenía febril. La IFI resultó positiva para $B$. henselae, a dilución de $1 / 256$, por lo cual se inició tratamiento antimicrobiano con ciprofloxacina, coincidiendo con la remisión de la fiebre. La paciente fue dada de alta al $30^{\circ}$ día de hospitalización, en buenas condiciones generales y sin dolor lumbar.

Caso clínico 2. Escolar de 10 años, sexo femenino, derivada del Servicio de Cirugía MáxiloFacial por masa submandibular, con 2 semanas de evolución, indolora, sin respuesta al tratamiento con amoxicilina. Se hospitalizó para estu- dio estando en buen estado general, afebril, con masa de $5 \times 5 \mathrm{~cm}$ submandibular derecha, dura, adherida a planos profundos, sin signos inflamatorios. El hemograma tenía 7.600 leucocitos/ $\mathrm{mm}^{3}$ y hematocrito de 33,2\%, PCR: $12 \mathrm{mg} / \mathrm{Lt}$. Una ecotomografía de tejidos blandos mostró un aumento de volumen sugerente de adenopatías, con aspecto de necrosis y de posible origen inflamatorio. Se realizó una punción para adenograma cuya muestra fue insuficiente. La IFI para $B$. henselae resultó positiva en dilución de $1 / 526$, iniciándose tratamiento con claritromicina e indicándose el alta al décimo día de hospitalización. Dos semanas después se realizó cintigrama óseo (dosis: 16 mCi ev de MDP-Tc99m, gammacámara Picker Dyna 15 unida a computador Alfanuclear) que mostró hipercaptación del MDPTc99m en la región posterior de la cresta ilíaca derecha (Figura 2), por lo que se prolongó el tratamiento antimicrobiano. La radiografía de pelvis y articulaciones sacroilíacas no mostraron alteraciones. En un control posterior se observó involución de la masa submandibular y el paciente estaba en excelente estado general, asintomática. Un segundo $\mathrm{CO}$ realizado 4 meses después del inicio del cuadro mostró regresión completa de la lesión descrita anteriormente (Figura 3).
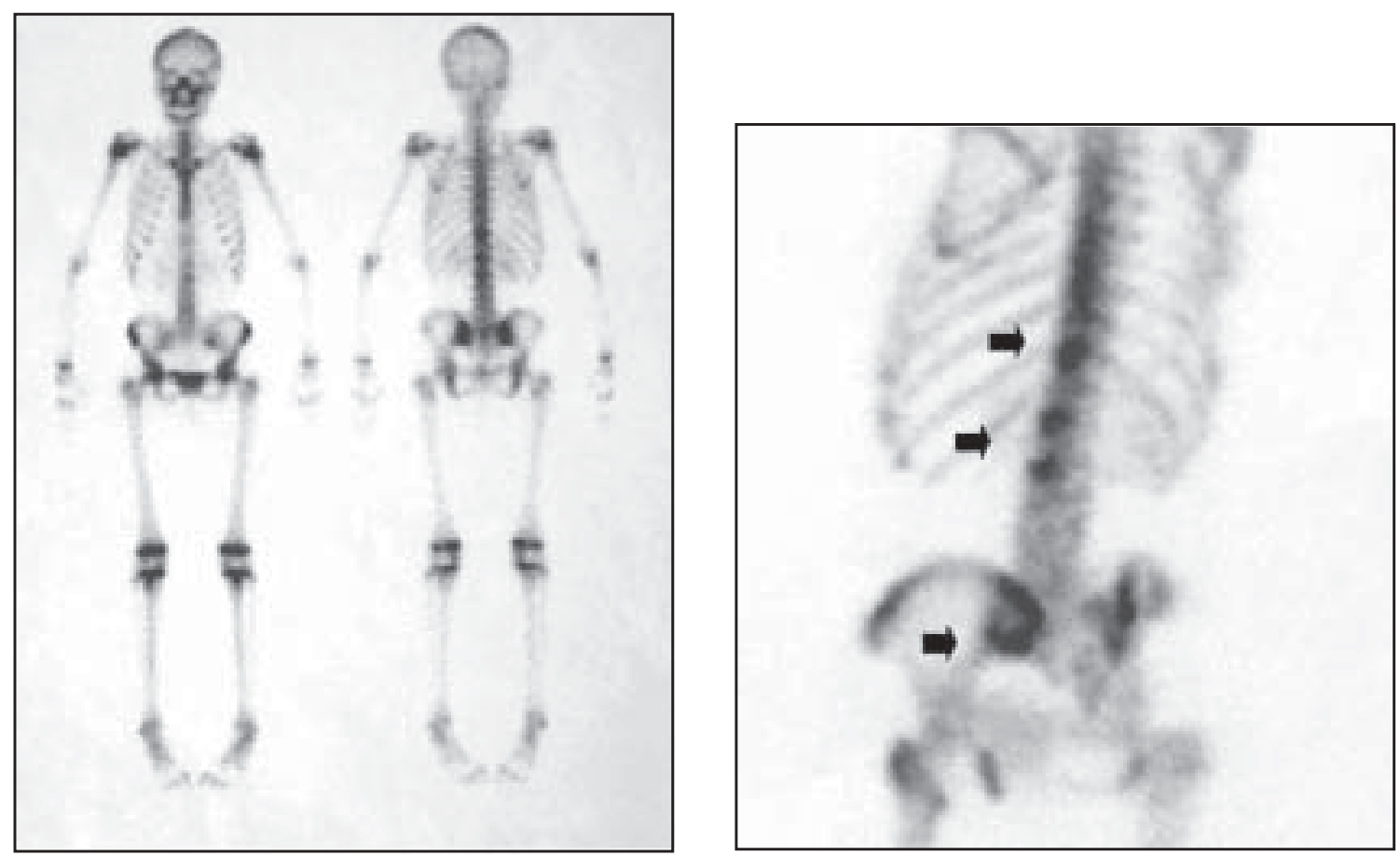

Figura 1. a. Cintigrama óseo mostrando lesiones en columna vertebral (D11, L1 y L2) y articulación sacroilíaca izquierda, evidentes en imágenes por posterior y anterior. b. Proyección oblicua posterior izquierda de columna y pelvis que permite localizar mejor las lesiones. Hay compromiso de los cuerpos vertebrales y región inferior de articulación sacroilíaca izquierda. 


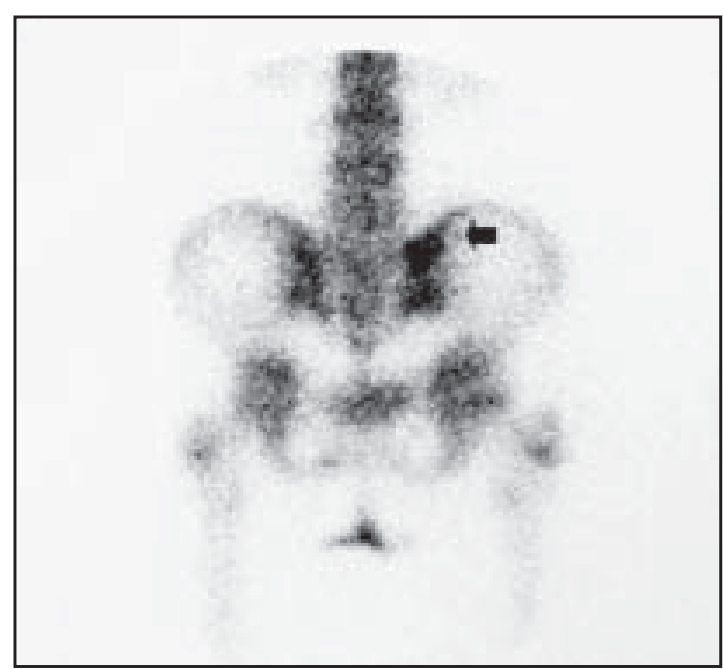

Figura 2. Imagen póstero-anterior de pelvis mostrando hiperfijación del $\mathrm{Tc}_{99 \mathrm{~m}}$-MDP en región más posterior de la cresta ilíaca derecha.

\section{Discusión}

De acuerdo a las series recopiladas en los últimos 10 años, la incidencia de osteomielitis y osteítis en la EAG es de aproximadamente 8\% (Tabla 1), cifra considerablemente más alta que la descrita por Carithers ${ }^{25}$ y por Margileth ${ }^{28}$ en las primeras grandes series referidas al tema hace unas décadas atrás. Esta diferencia puede ser debida en parte al mejoramiento de las pruebas diagnósticas de laboratorio y técnicas de imagen, que permiten un mejor estudio de las complicaciones sistémicas y naturalmente, a un mayor grado de sospecha del médico, conforme ha ido aumentando el conocimiento de la enfermedad. Sin embargo, no existen series recientes que hayan buscado en forma sistemática el compromiso óseo en pacientes con infección por $B$. henselae, independientemente de que tengan o no síntomas del aparato locomotor. Por lo anterior consideramos de interés el análisis de ambos casos.

En relación al primero, las manifestaciones de dolor abdominal probablemente se deban a la adenitis mesentérica; sin embargo, parte de los mismos pueden estar relacionados con dolor referido secundario al compromiso óseo de D11. La manifestación clínica en el esqueleto axial, sumado al síndrome febril y los reactantes de fase aguda, obligaron a la búsqueda de un compromiso infeccioso del aparato locomotor. Frecuentemente el dolor de las espondilo-discitis suele ser discreto o ausente en casos pediátricos. Sin embargo, el compromiso infeccioso de la

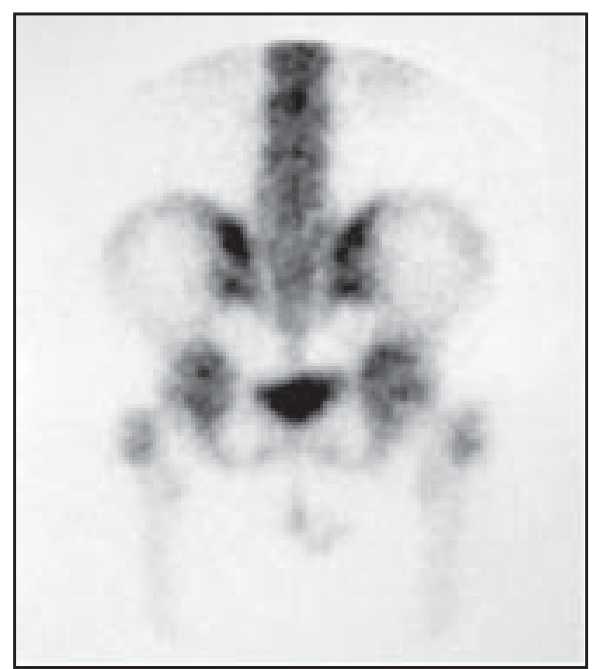

Figura 3. Mismo paciente de Figura 2 al cuarto mes de evolución del cuadro, con normalización del cintigrama óseo.

articulación sacroilíaca se caracteriza por lo intenso e invalidante de sus manifestaciones dolo$\operatorname{rosas}^{32}$. Dado el resultado serológico positivo y el curso evolutivo posterior favorable, no ameritó otro estudio.

El segundo caso es interesante dado que no presentó síntoma alguno referido al aparato locomotor ni un cuadro grave de fiebre y deterioro general, comúnmente asociados a la EAG atípica; y a que su seguimiento mostró normalización de las imágenes. Como se observó en esta niña, y también en otros casos publicados ${ }^{21,33}$, el compromiso óseo puede ocurrir tardíamente, semanas o meses después de la resolución clínica del cuadro.

Es importante destacar que el diagnostico final en ambos casos fue en base a las manifestaciones clínicas, apoyado por la serología y complementado por las imágenes.

De acuerdo a la literatura internacional y también a la experiencia nacional, las localizaciones óseas más frecuentes corresponden al esqueleto axial, particularmente columna dorsal, lumbar y pelvis, siendo igualmente uni o multifocal (Tabla 2). En los huesos largos el compromiso óseo puede localizarse igualmente en la diáfisis y en la metáfisis. El predominio en varones del compromiso óseo, observado en la mayoría de las series, ha sido explicado por factores conductuales como mayor agresividad en el contacto con sus mascotas, aumentando el riesgo de arañazos y mordiscos por el animal.

Aunque las lesiones producidas en el hueso por B. henselae son comúnmente líticas ${ }^{34,35,37,38,44,45}$, 
Tabla 1. Frecuencia descrita en la literatura de compromiso osteoarticular en la enfermedad por arañazo de gato

\begin{tabular}{rccccc}
\hline $\begin{array}{c}\mathbf{N}^{\mathbf{0}} \text { casos } \\
\text { EAG }\end{array}$ & $\begin{array}{c}\text { Rango edad } \\
\text { (años) }\end{array}$ & $\begin{array}{c}\% \\
\text { hombres }\end{array}$ & $\begin{array}{c}\mathbf{N}^{\mathbf{c}} \mathbf{c o n} \\
\mathbf{C O A}\end{array}$ & $\begin{array}{c}\text { \% con } \\
\text { COA }\end{array}$ & Referencia; año \\
\hline 160 & $67 \%<30$ & - & 0 & 0 & $26 ; 1954$ \\
83 & - & - & 0 & 0 & $27 ; 1960$ \\
115 & $80 \%<20$ & 54,8 & 0 & 0 & $13 ; 1968$ \\
1.200 & $9 \mathrm{~m}-84$ & 54,0 & 2 & 0,002 & $25 ; 1985$ \\
1.174 & - & - & 0 & 0 & $28: 1987$ \\
4 & $3-13$ & 50,0 & 1 & 25,0 & $29: 1995$ \\
9 & $2-13$ & 66,7 & 2 & 22,0 & $30 ; 1996$ \\
10 & $6-13$ & 70,0 & 0 & 0 & $31 ; 1996$ \\
7 & - & - & 0 & 0 & $20 ; 1998$ \\
15 & $5,5-13,5$ & 80,0 & 1 & 6,7 & $10 ; 1998$ \\
20 & $1,1-14,1$ & 70,0 & 1 & 5,0 & $32 ; 2000$ \\
41 & - & - & 0 & 0 & $16 ; 2000$ \\
20 & $1-14$ & 70,0 & 1 & 5,0 & $21 ; 2000$ \\
\hline
\end{tabular}

EAG: enfermedad por arañazo de gato, COA: compromiso osteoarticular.

la radiografía ha mostrado una baja sensibilidad en las etapas precoces de la enfermedad ósea $^{18,24,30,39,42,45}$. Debido a que estas lesiones se asocian también a cierto grado de actividad osteoblástica, predominantemente periférica a la zona lítica y pesquisable a veces con la radiografía en forma de esclerosis ${ }^{35,46}$, el CO es capaz de detectar de manera altamente sensible y precoz un proceso osteomielítico causado por $B$. henselae. Además del aumento de actividad osteoblástica, la mayor captación del MDP-Tc $\mathrm{c}_{99 \mathrm{~m}}$ por las lesiones visible en el $\mathrm{CO}$ puede estar relacionada con el importante componente inflamatorio y la necrosis central presente en la biop$\mathrm{sia}^{22,42,46}$. Esto, unido a la capacidad de este examen de explorar todo el esqueleto de una vez, hace que el CO sea irreemplazable en el estudio de complicaciones sistémicas óseas de estos pacientes.

Por otro lado, el CO es útil también en etapas prediagnósticas, en cuadros febriles o no, con sospecha de etiología neoplásica y particularmente infecciosa, especialmente si hay dolor óseo. En estos casos, puede ayudar en la pesquisa del compromiso óseo, y en caso de ser negativo, descartando con alto grado de seguridad la presencia de osteítis, osteomielitis, espondilo-discitis, artritis, metástasis óseas osteoblásticas y tumores óseos primarios. En el caso específico de la EAG, los diagnósticos diferenciales de un $\mathrm{CO}$ positivo corresponden principalmente a cuadros infecciosos, neoplásicos y traumáticos (Tabla 3). Si bien este examen es inespecífico, y comúnmente no permite conocer la etiología del cuadro, puede orientar el diagnóstico según las características de las lesiones, número, ubicación, intensidad de captación y extensión en el hueso.

Otros estudios imagenológicos como la $\mathrm{TAC}^{18,30,38,40}, \mathrm{RM}^{43}$ y Galio ${ }^{67,17,24,38,43}$ han sido empleados también con éxito en la detección de compromiso óseo en la EAG, permitiendo éste último además identificar lesiones en otros órga$\operatorname{nos}^{17} \mathrm{y}$ frecuentemente las linfoadenopatías asociadas a esta enfermedad ${ }^{30,43}$.

Si bien la EAG, por su carácter benigno y autolimitado, no requiere tratamiento antimicrobiano en su forma típica, cuando existen complicaciones sistémicas de la enfermedad, la mayor parte de los autores recomienda su uso. Esto es válido también en el caso de compromiso óseo por EAG, aún cuando no ha sido claramente demostrado que la terapia antimicrobiana modifique la evolución natural del cuadro.

Creemos de importancia realizar a futuro un estudio sistemático para determinar la real frecuencia del compromiso óseo; nuestra impresión es que es mayor de lo previsto y que seguramente como en otros cuadros infecciosos, sus expresiones clínicas, síntomas y signos predominantes deben estar influidos no sólo por factores del huésped, sino también por las características antigénicas del microorganismo. 
Tabla 2. Ubicación y número de lesiones esqueléticas descritos en la literatura

\begin{tabular}{|c|c|c|c|c|c|}
\hline $\begin{array}{l}\text { Edad } \\
\text { (años) }\end{array}$ & Sexo & $\begin{array}{c}\text { Compromiso } \\
\text { esquelético }\end{array}$ & Distribución & Ubicación & Referencia; año \\
\hline 5 & M & unifocal & axial & $\mathrm{P}(\mathrm{HI})$ & $35 ; 1954$ \\
\hline 4 & M & unifocal & apend & Fémur & $36 ; 1959$ \\
\hline 6 & $\mathrm{~F}$ & unifocal & apend & Metatarso & $37 ; 1969$ \\
\hline 2,5 & M & unifocal & axial & $\mathrm{E}$ & $38 ; 1983$ \\
\hline 18 & M & unifocal & axial & $\mathrm{Cd}$ & $39 ; 1985$ \\
\hline 25 & M & unifocal & axial & $\mathrm{Cd}(\mathrm{T} 10)$ & $40 ; 1986$ \\
\hline 9 & M & polifocal & axial & $\mathrm{Cr}, \mathrm{Cd}, \mathrm{Cl}, \mathrm{P}(\mathrm{ASI}$ y acetábulo) & $24 ; 1992$ \\
\hline 10 & $\mathrm{~F}$ & unifocal & axial & $\mathrm{Cl}(\mathrm{L} 2)$ & $41 ; 1992$ \\
\hline 12 & M & bifocal & axial & $\mathrm{Cd}$ (T5), PC & $42 ; 1993$ \\
\hline 4 & M & unifocal & axial & $\mathrm{Cd}$ & $18 ; 1994$ \\
\hline 9 & M & bifocal & axial-apend & Cr, clavícula & $34 ; 1994$ \\
\hline 11 & $\mathrm{~F}$ & unifocal & axial & $\mathrm{Cr}$ & $22 ; 1998$ \\
\hline 9 & M & unifocal & apend & Metacarpo & $11 ; 1998$ \\
\hline 10 & M & polifocal & axial & P (RIP, HI, isquion) & $43 ; 1998$ \\
\hline 4 & $\mathrm{~F}$ & bifocal & axial & $\mathrm{Cr}$ & $23 ; 1998$ \\
\hline 10 & M & unifocal & axial & $\mathrm{Cl}(\mathrm{L} 4)$ & $44 ; 1999$ \\
\hline 9 & M & bifocal & axial & $\mathrm{PC}$ & $45 ; 2000$ \\
\hline 10 & $\mathrm{~F}$ & polifocal & axial & $\mathrm{Cr}, \mathrm{PC}, \mathrm{Cc}, \mathrm{Cd}, \mathrm{Cl}, \mathrm{P}(\mathrm{HI})$ & $46 ; 2000$ \\
\hline 7 & $\mathrm{~F}$ & unifocal & apend & Húmero & $21 ; 2000$ \\
\hline 9 & M & polifocal & axial & $\mathrm{Cc}, \mathrm{Cd}, \mathrm{Cl}, \mathrm{P}$ (RIP) & $46 ; 2000$ \\
\hline 9 & $\mathrm{~F}$ & bifocal & axial & $\mathrm{Cl}, \mathrm{P}(\mathrm{HI})$ & $19 ; 2000$ \\
\hline 12 & M & bifocal & axial-apend & $\mathrm{Cd}$, Fémur & $19 ; 2000$ \\
\hline
\end{tabular}

M: sexo masculino; F: sexo femenino; Apend: esqueleto apendicular; ASI: articulación sacroilíaca; Cc: columna vertebral cervical; Cd: columna vertebral dorsal; $\mathrm{Cl}$ : columna vertebral lumbar; Cr: cráneo, E: esternón; HI: hueso ilíaco; $\mathrm{P}$ : pelvis; PC: parrilla costal; RIP: rama isquiopubiana.

\section{Conclusiones}

- Cuando en un paciente en estudio por un síndrome febril de origen desconocido, el CO demuestre compromiso esquelético axial uni o multifocal, debe plantearse la posibilidad de una EAG atípica entre las etiologías benignas que pueden causar dicho aspecto cintigráfico que, como se sabe, puede también ser causado por otras patologías de origen infeccioso, traumático o neoplásico.

- En pacientes en los cuales se diagnosticó una EAG podría ser de interés documentar el posible compromiso óseo mediante la realización de un $\mathrm{CO}$, especialmente en aquellos con un cuadro clínico de mayor gravedad, en que el dolor óseo puede ser encubierto por otros síntomas más severos de la enfermedad. Este examen es útil aún en pacientes sin dolor óseo y aquellos sintomáticos con radiografías normales.
Tabla 3. Diagnósticos diferenciales cintigráficos principales de enfermedad por arañazo de gato con compromiso óseo en población pediátrica

Neoplasias con compromiso óseo
Leucemia
Neuroblastoma
Histiocitosis
Linfoma
Tumores óseos primarios
Sarcoma de Ewing
Osteosarcoma
Enfermedades infecciosas
Osteomielitis aguda
Osteomielitis crónica
Espóndilo-discitis
TBC ósea
Lesiones traumáticas
Fracturas
Fracturas de stress
Maltrato infantil
Otras
Displasia fibrosa




\section{Resumen}

La enfermedad por arañazo de gato (EAG) es una patología benigna y autolimitada transmitida por el rasguño o mordedura de un gato portador de Bartonella henselae, que se manifiesta como linfoadenopatía cercana al sitio de inoculación y que afecta, en $80 \%$ de los casos, a sujetos bajo 21 años de edad. Menos de $25 \%$ de los pacientes desarrollará la forma atípica de la enfermedad, con compromiso de órganos como hígado, bazo, sistema nervioso central y retina. La osteomielitis ha sido descrita en series antiguas como de escasa ocurrencia, aunque publicaciones recientes sugieren una incidencia mayor. El esqueleto axial es el más frecuentemente comprometido, principalmente pelvis y columna dorsolumbar, pudiendo ser las lesiones esqueléticas únicas o múltiples; éstas no siempre son dolorosas y pueden aparecer hasta semanas o meses después del inicio de los síntomas. Se presenta los casos clínicos de dos niñas con compromiso esquelético, serología positiva para $B$. henselae y recuperación completa posterior. Una de ellas (edad 12 años) presentó fiebre y dolor abdominal severo, demostrándose lesión hipodensa hepática y osteomielitis multifocal en la columna y pelvis. La otra (edad 10 años) sólo cursó con adenopatía submandibular, sin fiebre ni compromiso general, y una lesión indolora en la cresta ilíaca, visible al cintigrama óseo (CO). Un $\mathrm{CO}$ con lesiones axiales uni o multifocales en un paciente febril sin etiología clara, debe hacer sospechar EAG atípica entre las posibles causas. El CO es indispensable si se quiere descartar un compromiso óseo asociada a EAG, especialmente en pacientes sintomáticos con radiografías normales e incluso, aquellos sin dolor óseo.

\section{Bibliografía}

1.- Miranda M. Diagnóstico diferencial de las enfermedades reumatológicas según edad. Reumatología 1999; 15: $110-1$.

2.- Sonnen G M, Henry N K. Pediatric bone and joint infections: diagnosis and antimicrobial management. Pediatr Clin North Am 1996; 43: 933-47.

3.- Shetty A, Gedalia A. Septic arthritis in children. Rheumatol Dis Clin North Am 1998; 24: 287-304.

4.- Wear D J, Margileth A M, Handfield T L, Fischer G W, Schlagel C J, King F M. Cat scratch disease: a bacterial infection. Science 1983; 221: 1403-5.

5.- Bass J W, Vincent J M, Person D A. The expanding spectrum of Bartonella infections: II. Cat-scratch disease. Pediatr Infect Dis J 1997; 16: 163-79.

6.- Raoult D. Bartonella infection in humans. Press Med 1999; 28: 429-34, 438.

7.- Parrochia E. Gatos y ratas: rasguños y mordeduras.
Bol Hosp S J de Dios 1998; 45: 99-102.

8.- Wolff M. Cambios epidemiológicos en las enfermedades infecciosas en Chile durante la década 1990-2000. Rev Méd Chile 2002; 130: 353-62.

9.- Pimont Y, Heller R. Bartonellosis: I. Bartonella henselae. Ann Biol Clin (Paris) 1998; 56: 681-92.

10.- Karpathios T, Golphinos C, Psychou P, Garoufi A, Papadimitriou A, Nicolaidou P. Cat scratch disease in Greece. Arch Dis Child 1998; 78: 64-6.

11.- Keret D, Giladi M, Kletter Y, Wientroub S. Cat-scratch disease osteomyelitis from a dog scratch. J Bone Joint Surg Br 1998; 80: 766-7.

12.- Tsukahara M, Tsuneoka H, Iino H, Ohno K, Murano I. Bartonella henselae infection from a dog. Lancet 1998; 352: 1682.

13.- Margileth A M. Cat scratch disease: nonbacterial regional lymphadenitis. The study of 145 patients and review of the literature. Pediatrics 1968; 42: 803-18.

14.- Anderson B E, Neuman M A. Bartonella spp. as emerging human pathogens. Clin Microbiol Rev 1997; 10: 203-19.

15.- Schutze G E. Diagnosis and treatment of Bartonella henselae infections. Pediatr Infect Dis 2000; 16: 11858.

16.- Tsukahara M, Tsuneoka H, Iino H, Murano I, Takahashi H, Uchida M. Bartonella henselae infection as a cause of fever of unknown origin. J Clin Microbiol 2000; 38: 1990-1.

17.- Wong M, Isaacs D, Dorney S. Fever, abdominal pain and an intracranial mass. Pediatr Infect Dis J 1995; 14: $725-8$.

18.- Koranyi K. Fever, back pain and pleural effusion in a four-year-old boy. Pediatr Infect Dis J 1994; 13: 671-3.

19.- Ladrón De Guevara D, Lobo G, Wu E et al. Resultado del cintigrama óseo en 37 pacientes pediátricos con síndrome febril prolongado: evaluación retrospectiva. Bol Hosp S J de Dios 2000; 47: 101-9.

20.- Jacobs R F, Schutze G E. Bartonella henselae as a cause of prolonged fever and fever of unknown origin in children. Clin Infect Dis 1998; 26: 80-4.

21.- Massei F, Messina F, Talini I et al. Widening of the clinical spectrum of Bartonella henselae infection as recognized through serodiagnosis. Eur J Pediatr 2000; 159: 416-9.

22.- Berg L C, Norelle A, Morgan W A, Washa D M. Catscratch disease simulating histiocytosis X. Hum Pathol 1998; 29: 649-51.

23.- Gaebler J W, Burgett R A, Caldemeyer K S. Subacute orbital abscess in a four-year-old girl with a new kitten. Pediatr Infect Dis J 1998; 17: 844-6.

24.- Cohen-Abbo A, Cheatham S, Edwards K. Disseminated cat-scratch disease simulating neuroblastoma. Pediatr Infect Dis J 1992; 11: 1058-60.

25.- Carithers H A. Cat-scratch disease. An overview based on a study of 1200 patients. Amer J Dis Child 1985; 139: 1124-33.

26.- Daniels W B. Cat scratch disease. Report of one hundred sixty cases. JAMA 1954; 154: 1247-51.

27.- Spaulding W B, Hennessy J N. Cat scratch disease. A study of eighty-three cases. Amer J Med 1960; 28: 504-9.

28.- Margileth A M, Wear D J, English C K. Systemic cat scratch disease: report of 23 patients with prolonged or recurrent severe bacterial infection. J Infect Dis 1987; 155: 390-402.

29.- Sölder B, Allerberger F, Covi B et al. Cat scratch disease caused by Bartonella henselae. Immun Infekt 1995; 23: 228-31. 
30.- Hopkins K L Simoneaux S F, Patrick L E, Wyly J B, Dalton M J, Snitzer J A. Imaging manifestations of Cat-Scratch Disease. AJR 1996; 166: 435-8.

31.- Abarca K, Vial P A, Rivera M et al. Infección por Bartonella henselae en pacientes inmunocompetentes: Enfermedad por arañazo de gato. Rev Méd Chile 1996; 124: $1341-9$.

32.- Toso Ms, Norambuena X. Sacroileítis infecciosa en niños. Reumatología 1998; 14: 94-6.

33.- Waldvogel K, Regnery R L, Anderson B E, Caduff R, Caduff J, Nadal D. Disseminated cat-scratch disease: detection of Rochalimaea henselae in affected tissue. Eur J Pediatr 1994; 153: 23-7.

34.- Adams W C, Hindman S M. Cat-scratch disease associated with osteolytic lesion. J Pediatr 1954; 44: 665-9.

35.- Collipp P J, Koch R. Cat-scratch fever associated with an osteolytic lesion. N Engl J Med 1959; 260: 278-80.

36.- Carithers H A, Carithers C M, Edwards R O Jr. Catscratch disease: its natural history. JAMA 1969; 207: 312-16.

37.- Carithers H A. Cat-scratch disease associated with an osteolytic lesion. Am J Dis Child 1983; 137: 968-70.

38.- Johnson J F, Lehman R M, Shiels W E, Blaney S M. Osteolysis in cat-scratch fever. Radiology 1985; 156: 373-4.
39.- Gregory D W, Decker M D. Case report: cat scratch disease: an infection beyond the lymph node. Am J Med Sci 1986; 292: 389-90.

40.- Hulzebos C V, Koetse H A, Kimpen J L L, Wolfs T F W. Vertebral osteomyelitis associated with cat-scratch disease. Clin Infect Dis 1999; 28: 1310-2.

41.- Fretzayas A, Tapratzi P, Kavazarakis E, Sinaniotis C. Multiorgan involvement in systemic cat-scratch disease. Scand J Infect Dis 1993; 25: 145-8.

42.- Ratner L M, Kesack A, Mccauley T R, Disler D G. Disseminated Bartonella henselae (cat-scratch disease): appearance of multifocal osteomyelitis with MR imaging. AJR 1998; 171: 1164-5.

43.- Larsen C E, Patrick L E. Abdominal (liver, spleen) and bone manifestations of cat scratch disease. Pediatr Radiol 1992; 22: 353-5.

44.- Täger M, Zamorano J. Osteomielitis, una manifestación inusual de la enfermedad por arañazo de gato. Rev Chil Infect 2000; 17: 326-31.

45.- Wolff E, Muñoz M P, Zapata C, Ledermann W. Enfermedad por arañazo de gato complicada con compromiso sistémico, osteomielitis osteovertebral y absceso paravertebral. Rev Chil Infect 2000; 17: 332-9.

46.- Muszynski M J, Eppes S, Riley H D. Granulomatous osteolytic lesion of the skull associated with cat-scatch disease. Pediatr Infect Dis J 1987; 6: 199-201.

Correspondencia a:

David Ladrón de Guevara $\mathrm{H}$.

E-mail: humdavidhm@hotmail.com 